\title{
Human-Centric Functional Modeling and Evolutionary Biology
}

\author{
Andy E. Williams ${ }^{1 *}$ \\ ${ }^{1}$ Nobeah Foundation, Nairobi, Kenya \\ *awilliams@nobeahfoundation.org
}

\begin{abstract}
The newly emerging science of Human-Centric Functional Modeling provides an approach towards modeling biological and other systems that is hypothesized to maximize human capacity to understand and navigate complexity in those systems. This paper provide an overview exploring how HumanCentric Functional Modeling might be applied in evolutionary biology, and how this increase in capacity to understand the complexity that organisms have evolved into might be achieved. The broader usefulness of Human-Centric Functional Modeling is that it provides a simple mathematical definition of what constitutes a biological system, defines the problem-solving domain of any biological system in terms of abstract mathematical spaces, and provides an expression defining general problem-solving ability in any such domain. This enables it to be seen that all systems with general problem-solving ability in their own domain are potentially an abstraction of a single mathematical pattern of adaptive problem-solving that might apply to all domains. From this perspective nature has already potentially solved problems in biological organisms that can be represented in some abstract functional state spaces as the same general problem that must be solved to address problems in a wide range of other systems, including existential challenges from poverty to climate change, where Human-Centric Functional Modeling enables it to be seen that not only can nature's solutions be copied, but that nature has demonstrated its solutions to have worked for hundreds of millions of years.
\end{abstract}

\section{Introduction}

In Human-Centric Functional Modelling living systems such as the human organism are modelled as having a set of observable behaviours (functions) through which they transition from one functional state to another. These states form a "functional state space" which the system acting in that domain moves through. All the states accessible through those functions (i.e. all the states in the "functional state space") then reflect all possible behaviors of the system.

\section{Representing Organisms as Networks}

From the perspective of Human-Centric Functional Modeling, any system within an organism has behavior confined to a functional state space. For example, the cognitive system is represented as navigating a space of concepts or a "conceptual space" (the functional state space of cognition). All possible behaviors of any system are confined to the states accessible within a given domain of behaviour (i.e. all states belong to some common category defined by the domain object that classifies every functional state in the space, where in the case of the cognitive system the common domain object or category is the "concept"). These functional state spaces are described in terms of a graph containing a network of nodes representing the functional states, where those states are connected by and interact through edges representing transition processes between those states. In the case of the cognitive system these transition processes are the reasoning processes through which the cognitive system transitions from one concept to another. It has been hypothesized that all complex systems can be represented by such networks [1]. If so, then living processes from homeostasis, reproduction, growth, and multicellular cooperation, all the way up to cognition and consciousness can potentially be represented as processes that navigate their own functional state spaces described by such networks. The presence of such a common structure or pattern related to networks that potentially underlies all 
life is hinted at by research suggesting that "rapid advances in network biology indicate that cellular networks are governed by universal laws" [3], and the potential impact of this approach in uncovering any such structure or pattern is found in statements that such networks offer "a new conceptual framework that could potentially revolutionize our view of biology and disease pathologies in the twenty-first century" [3].

Any system with a stable set of repeatable functions also must stay within a bounded region of a "fitness space" that describes the fitness of the system to execute its functions. A change in fitness of the system occurs as a result of some action, that is, as a result of following some path in functional state space. What constitutes a fitness space, and what constitutes the most generalized fitness space possible is a lengthy discussion pursued elsewhere through ongoing work on the subject of a "Semantic Metrics Framework" for measuring the relative fitness of any function in achieving generalized outcomes [2]. In any case, defining a generalized "fitness space" for all problems, such as defined by the three dimensions of target value of fitness, actual value of fitness, and predicted value of fitness proposed within the Semantic Metrics Framework, then the path through this fitness space must stay within a bounded region of that fitness space. Any living process is represented as some algorithm that chooses a path through functional state space which results in a path through fitness space that is dynamically stable. In this sense the motion in fitness space must be stable globally throughout the fitness space, despite potentially being chaotic in functional state space due to random interactions with the environment. At least one set of equations, the Lorenz equations for convection, display this stability in three dimensions as required. Because this pattern of stability potentially applies to all life processes at all scales, it is scale-invariant and might conceivably give rise to fractal properties in organisms [11], [12].

Homeostasis or other processes of adaptation might be seen as transmitting information about the fitness of a given behavior in achieving that adaptation, and therefore might be governed by information theory [6] in the sense that if the channel is too noisy then the signal of fitness cannot be resolved and adaptation cannot occur. However, some noise at the boundaries of the system, that is, some behavior outside the boundaries of the system, is always expected in natural processes such as adaptation. Whenever enough of the behavior of a system within an organism goes outside of the boundaries of the category of functional state defining that original system, it might eventually emerge into some new domain to create a new system. If so, since those new behaviors are perturbations of the behaviors of the old system, they would be expected to be sensitive to that initial system, and therefore to be able to interact with that system despite being outside of it. The behavior of a system goes outside its boundaries to form a new system when the numbers of new behaviors, and the differentiation between those behaviors and the behaviors in the original domain increase until they form another domain of repeatable behavior, which is one definition of another system. There are at least two ways that some interaction between the new and original systems might occur. One is that these systems could form a hierarchy through which the newer system could interact with the older one. An example is the tactile system which can be seen as creating tactile sensations that interact with the awareness's of the consciousness system higher up in the hierarchy of the human organism. Another way these two systems could interact is that the newer system could simply create a parallel copy of the older one. The two would then interact though one copying the infrastructure of the original without the functional states interacting directly. An example is the visual system which can be seen as copying the tactile system to "touch" light, without the sense of sight interacting with that of touch.

New systems that occupy a hierarchical position with respect to the original system might also copy infrastructure from the original. Assume that all the potentially complex behaviors in a given domain can be represented as some combination of a very simple set of behaviors (functions or operations). In 
other words, assume that any network of functional states can be constructed with some sequence of only a few different types of operations. In mathematical terms a set of operations is said to "span" a space when any point in that space can be represented as some combination of those operations. It has been hypothesized that a set of four operations can "span" the network of functional states representing conceptual space [14]. Representing the mind as moving through its own functional state space (again, a space of concepts or a "conceptual space"), this means that a set of four operations can be used to represent any reasoning process in this space. This means that any reasoning required to navigate from one concept in conceptual space to another can be constructed as a composition of four functions [1]. Like the conceptual space, the awareness space navigated by the consciousness is also an "open" (unbounded) space. For this reason the same four functions might span the awareness space as well (with awarenesses as their input arguments and outputs rather than concepts), since it has been hypothesized that a set of four operations can "span" any open (unbounded) network of functional states. Therefore the same generalized functional components required to implement any such four functions might be reused in the consciousness system to span the awareness space. Whether systems like the emotions, or the body that might be represented as navigating "closed" functional state spaces are spanned in the same way, and whether such functions can be represented as having been copied from them or earlier biological processes, even going back to homeostasis, remains to be investigated.

In any case, assume that all the potentially complex behaviors in a given domain can be represented as some combination of a very simple set of behaviors (functions or operations) and that that any network of functional states can be constructed with some sequence of only a few different types of functional components implementing those operations. We can confirm that our cognition can interact with our perception of physical sensations or emotions because it can conceptualize them. We can confirm that our consciousness can interact with our perceptions of physical sensations, emotions, or concepts, because we can become aware of them. Consider tactile sensations to arise from signals originating from each sensory receptor in the array of all sensory receptors. Together these represent a virtual field of signals in three dimensions that varies with time. Representing physical sensations and emotions as such three dimensional fields, and representing each specific physical sensation or emotion as a specific signal in those fields, we can observe that our consciousness and cognition are able to detect signals at specific points in those fields. While four functions might be required for a system to interact with and therefore detect a functional state within its own functional state space, by our definition of a system, that system can't use those functions to detect functional states outside its own domain. Instead it must use functions that represent that other domain in different terms such as signals. It is hypothesized that only three functions are required for a system to be able to detect and interact with a signal representing any functional state in a different domain.

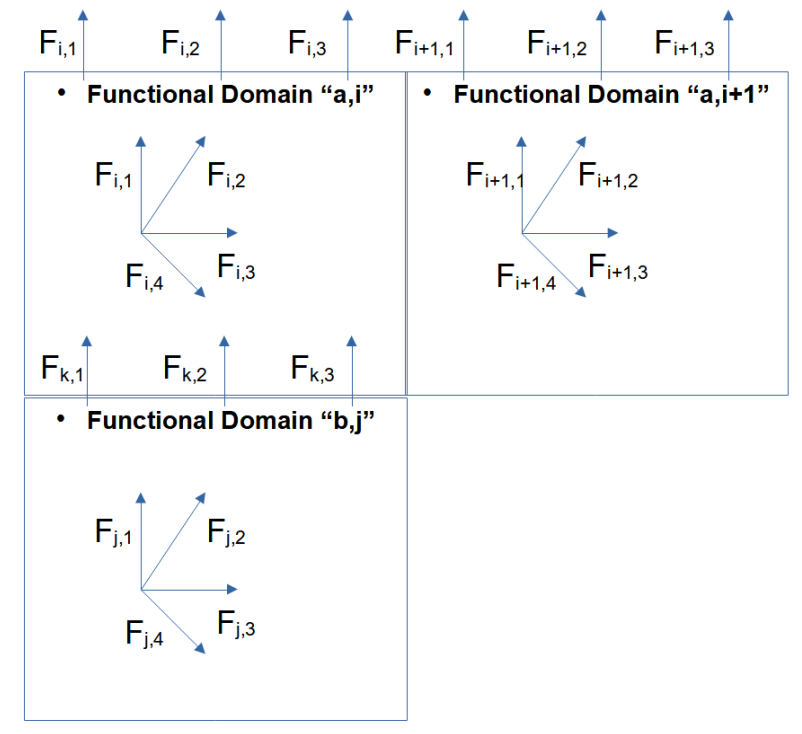


Figure 1: Case in which each system has four functions through it might navigate between the different functional states within its domain, and in which each system has three functions through which it might navigate between signals representing functional states in other functional domains. Through these three functions a higher (parent) system is able to interact with a lower (child) system across the boundary of functional domains. Sibling systems indicated by " $i$ " and " $i+1$ " do not interact.

The cognition is able to interact with (that is, to conceptualize) any sensory or emotional signal in those fields. Representing concepts as forming such a field as well, it is hypothesized that the same three functions can be used by the consciousness to interact with (to become aware of) any conceptual signal in that field, in the same way that it might use those same three functions to become aware of a signal within the five senses, or to become aware of an emotion. The point is that the same mechanism by which systems might interact might work between any two systems, and therefore might work if copied from one system to the next.

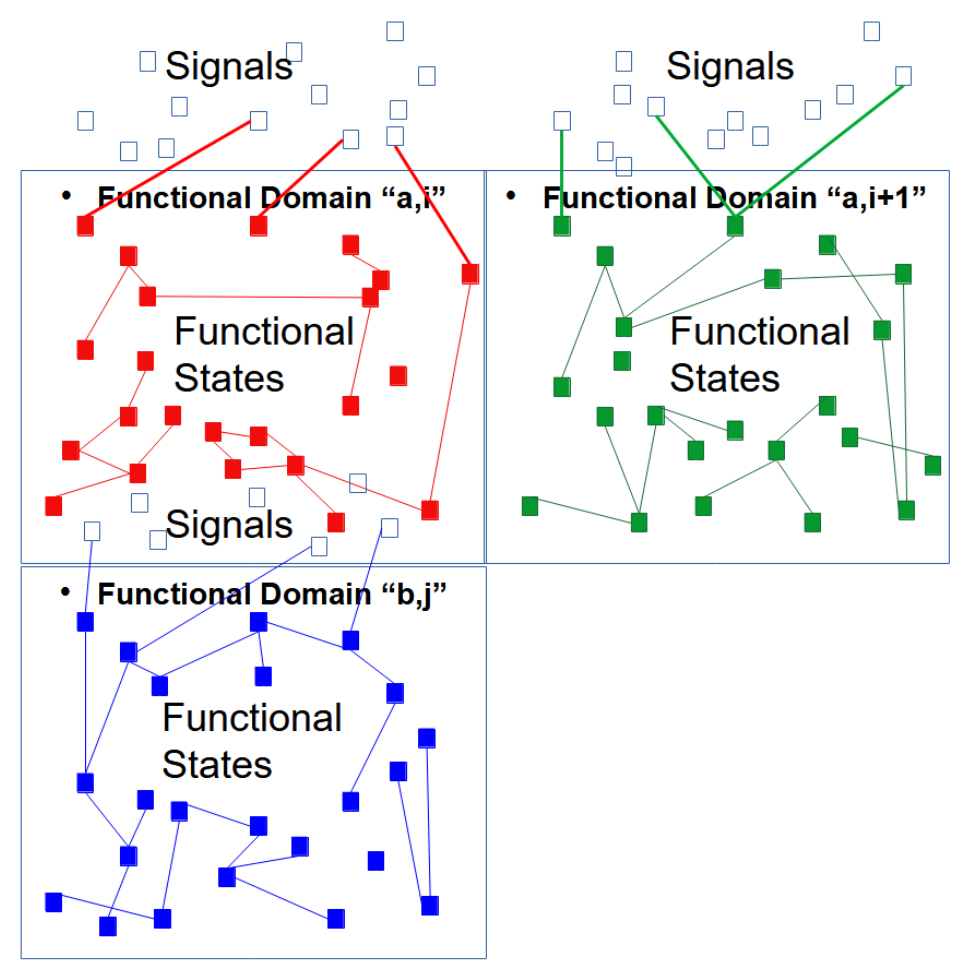

Figure 2: Interact with functional states in current domain and signals from other functional domains.

All biological systems in an organism, like the sensory-motor space, the emotional space, the conceptual space, and the awareness space, are hypothesized to form a hierarchy that exchange information. Since every functional state space is a network of nodes, then if every biological system can be represented this way, and if every organism consists of a hierarchy of biological systems, then from the perspective of Human-Centric Functional Modeling, every biological organism can potentially be represented at all scales as a hierarchy of interacting networks in which each network represents a system that is dynamically stable in some generalized fitness space. The importance of this scaleinvariance is that from the perspective of Human-Centric Functional Modeling, as few as a single set of equations is needed to achieve this dynamical stability in all processes of life. From this perspective, all living processes are adaptive problem-solving processes that solve the same problem of maintaining dynamical stability in a given domain through achieving general problem-solving ability in that 
domain. The usefulness of a single universal representation for systems in terms of their motion through some functional state space is that it provides a framework for understanding systems [13] that enables problems defined for a system in one domain, or solutions discovered for the system in that domain, to be generalized and applied to systems in potentially all other domains.

From this perspective Human-Centric Functional Modeling is a new method of biomimicry that might be used to increase the social impact of diverse disciplines like engineering and science through representing biological systems in terms of functional state spaces, which enables it to be seen that nature has already solved problems that can be represented in these abstract spaces as being relevant to a wide range of science and engineering disciplines, as well as relevant to social and organizational outcomes. Namely, achieving general problem-solving ability in any given domain is the same general problem that must be solved to address existential challenges from poverty to climate change, where nature has demonstrated those solutions to work for hundreds of millions of years. This insight that biologically inspired engineering design requires understanding of complex biological systems for use as analogues in engineering designs is not new [9]. In fact this insight is shared by others who suggest that "the architectural features of molecular interaction networks within a cell are shared to a large degree by other complex systems, such as the Internet, computer chips or society", and who suggest that "this unexpected universality suggests that similar laws govern the development and function of most complex networks in nature" [3].

As one example of the application of this biomimicry, AI safety can potentially be represented as a problem that nature has already solved [18]. Human-Centric Functional Modeling can be used to define AI safety as the problem of creating the capacity to develop a path through the "collective conceptual space" representing the collective reasoning processes of mankind, or through the collective "human state state space" representing the collective physical, emotional, and cognitive behavior of mankind, where the complexity of that path can reliably be increased to the point that it is greater than the complexity of the path that can be developed by a super-intelligent AI. Since the complexity an AI is capable of is not known, this reduces to the problem of creating the capacity to increase the complexity of collective reasoning or of increasing the complexity of behavior in the collective human state space, to an arbitrarily high degree. Representing the complexity of any solution in any functional state space as the length of the path corresponding to the solution within that space multiplied by the linear density of functional states the solution passes through, this might be equivalent to problems that nature has already solved, such as the problem of creating a greater capacity within the organism to respond to threats like viruses, than those threats typically pose, so that the natural balance is for species not to go extinct. One general pattern of solution is to scale cooperation where collective problem-solving ability scales with that cooperation [18].

\section{Purpose: Assessing the Validity of the Approach}

Given that representing systems in evolutionary biology is only one of a great many applications of Human-Centric Functional Modeling currently being explored, the goal of this paper is not to engage in exhaustive study to determine the validity of that representation, but to simply probe the issues to gain some assurance that the questions are valid and also that they have intellectual merit and broader implications, so that those questions might be opened for exploration by other specialists in this discipline. The goal is to create sufficient mind share such that collaborations might be formed to enable groups of local researchers to take part in a set of planned Large Scale international Collaborative REsearch (LSCORE) initiatives intended to test and to elaborate the underlying theory and to build components of the General Collective Intelligence infrastructure [15] required to achieve an exponentially greater impact on research [14] in evolutionary biology through Human-Centric Functional Modeling. Applying HCFM potentially involves a massive effort beginning with mapping 
out the mathematical space representing all possible behaviors of the cognitive system because the states of the cognitive system are the only ones which by definition we can be assured we can conceptualize, and then extending to efforts towards mapping out the mathematical spaces representing other systems the cognition interacts within this human organism, such as the sensory-motor system, the emotional system, and the consciousness system, and finally using the knowledge gained to map out other spaces related to biological processes such as homeostasis, or cellular reproduction. Using HCFM this effort might be approached methodically, dividing up these functional state spaces into equal geometries so that tens of thousands of scientists across many disciplines might be organized to map them out. Similar to the case of sequencing the human genome which provided a catalog that might make it much easier to establish connections between particular genes and disease, a map of the human organism providing such a catalog of processes might make it much easier to establish connections between particular functional constructs and physical, emotional, cognitive, or conscious wellness or disease.

\section{Adaptive Problem-Solving Systems Must Give Rise to Self-Similar Adaptive Problem-Solving Systems}

Whenever the behavior within a particular system goes outside that system, there is still some element of behavior that is within the system. As mentioned, one consequence is that behavior outside the system would be expected to still be able to interact with the system through being able to detect some information about the state of that system. As the number of behaviors outside the first system increases, some minimal set of those new behaviors might come to span a new functional state space. At the point where a set of new behaviors spans a new functional state space, the number of behaviors in the domain of that new functional state space is expected to increase exponentially [17]. As a result the entity might gain the ability to respond to an exponentially greater number of states of the external environment whenever new behaviors emerge into a system. At this point, wherever that system might have been limited to responding linearly to any change in the external environment, that limitation can potentially disappear through the emergence of the new system. As an example, in an inanimate system the change in temperature $\Delta \mathrm{T}$ is linearly related to the heat $\mathrm{Q}$ that has been added to the system, divided by the heating coefficient $\mathrm{c}$ and the mass $\mathrm{m}$ :

$$
T=\frac{Q}{c m}
$$

In the case of living systems however, there might be an exponentially greater number of processes through which the system might be able to react to the addition of thermal energy rather than just by heating up. For all external states of the environment that the new system can respond to, the system can potentially maximize production of entropy through the same cycle of dynamic stability as the original adaptive problem-solving system that gave rise to it [7]. The category of behavior in this new domain becomes an "emergent property" of the entity.

When perturbations to the adaptive problem-solving system are large enough and continue long enough, it is hypothesized to become probable that they must result in the development of a new system with it's own new functional state space. That new system might be adjacent to the original like the five sensory systems are adjacent (auditory, tactile, gustatory, olfactory, and visual systems) if the functional states are non-overlapping, or that new system might occur at a higher point within a hierarchy of systems (like consciousness or cognition as compared to the five sensory systems) if the functional states of the new system are more abstract and include the functional states of the previous system. 


\section{Adaptive Problem-Solving Systems Must Exceed Their Boundaries}

As mentioned, in Human-Centric Functional Modeling a "system" is defined as having behavior that is confined to a single functional state space in which all behaviors map from and map to a domain object in the same category. Since all functional states are represented by that same object within the functional domain in which the system operates, these states together form a space. For example, in HCFM the set of concepts navigated by the cognitive system form a conceptual space. If functional states are continually being created, there will be some finite probability that those new states might exist partially outside the functional state space of the current system. If any network of functional states can be constructed with some sequence of operations in a small set that "spans" the space, then accessing any states outside that space will require changes to those functions.

Because systems as defined by Human-Centric Functional Modeling are dynamically stable, perturbations in the behavior of one system are likely to give rise to a child or sibling system if the perturbations in that initial system are large enough and continued long enough that a sufficient range of new behaviors is explored. If the initial system has behavior that is spanned by some minimal set of functions, and if each of those functions experiences a large enough range of perturbations for a sufficient length of time such that their behavior overlaps a region in state space that defines a new domain object, then due to the stability of such systems the tendency for adaptive problem-solving functionality to remain in that new space to form a new child or sibling system might be the most stable outcome. Any function that exits the original domain might act on a different object than other functions that exit the original domain. Exiting functions need not overlap. The question is when is the overlap of functions into a new domain object large enough and stable enough to give rise to a new parent or sibling system?

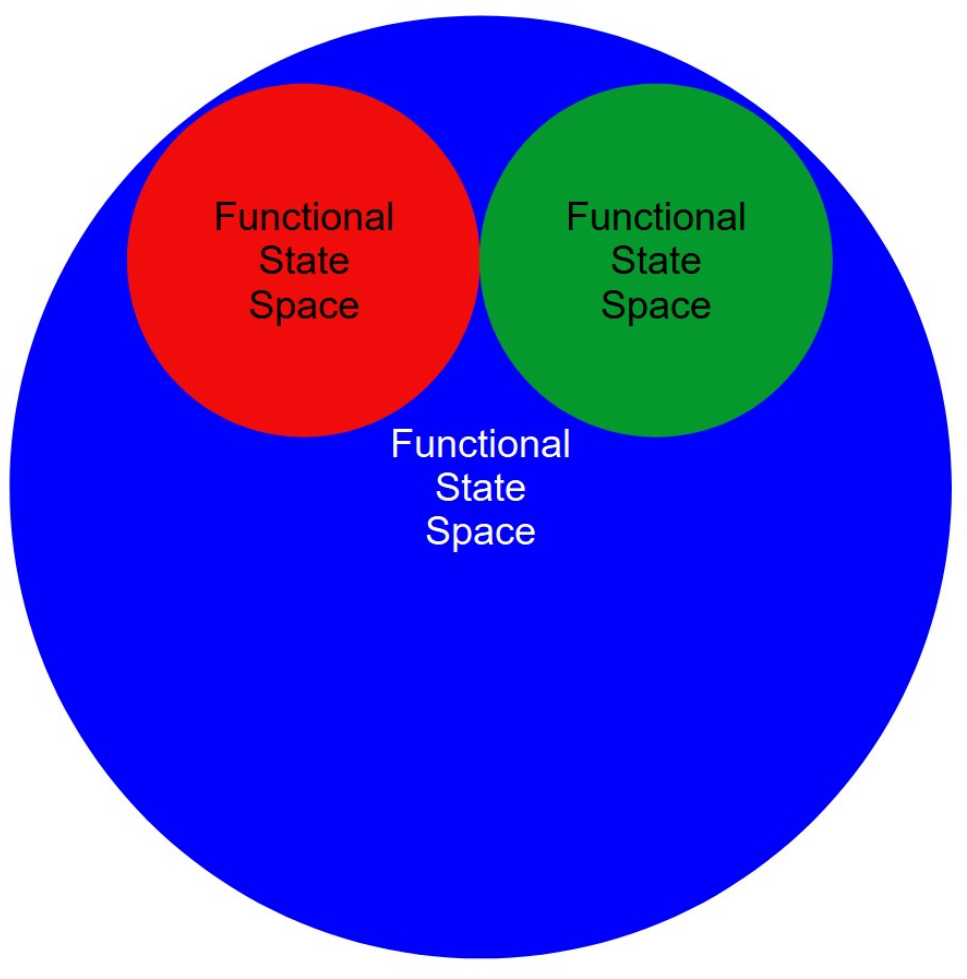


Figure 3: Non-overlapping functional states (red and green) and overlapping functional state (blue) that might be created by a new "system".

If a functional state of an entity is associated with lower potential energy than its current state, then the laws of thermodynamics ensure that entity will tend to transition to that lower energy state. If the functional states of that entity form a "system" in some domain, and if the functional states in that system are lower energy than the current state, then the entity would be expected to naturally tend towards occupying that system. If the volume of lower energy states in the functional state space of a system is exponentially greater at the emergence of a new system as expected, that exponentially greater volume of lower energy states in the functional state space of a new system suggests that any such new system forms a stable potential energy well that entities might occupy. Furthermore, cyclic processes maximize the production of entropy [17], [18]. The fact that energy is continually being added to such systems combined with the requirement that the dynamics of adaptive problem-solving systems be globally stable in some fitness space suggests that those dynamics are cyclic. What emerges is again a picture of adaptive problem-solving systems as cyclic processes that exist at all scales within organisms.

\section{Modeling the Implementation and Evolution of Biological Systems}

If every organism can be modeled as a hierarchy of networks, in which each biological system in that organism is represented as navigating a functional state space, and in which each biological process in that organism is represented by a path from one functional state to another, then every biological process in every organism can be mapped to a specific path in the functional state space within a specific level of that hierarchy of networks. Every functional state and every path between them in this hierarchy of networks then together potentially provides a complete index or "primary key" in database terms, for all functional models of all biological processes. Just as in the case of the cognitive system, understanding problems in terms of paths between functional states is far more straightforward than understanding problems in terms of potential models for how those processes might be implemented. How it might be possible to express the path of a given reasoning process from one concept to another in terms of biochemical molecules or any inputs and outputs other than the functional states (in this case concepts) native to the domain might not be readily understandable. But this mapping to between functional models and real implementations is also a problem this functional approach can help solve as well. This is a useful problem to solve. As stated by others "a major challenge of contemporary biology is to embark on an integrated theoretical and experimental programme to map out, understand and model in quantifiable terms the topological and dynamical properties of the various networks that control the behavior of the cell" [3]. The complexities that might be encountered remain to be discovered and might be significant, since it has been suggested that mapping out these interactions between such dynamically changing networks is fundamentally distinct from mapping out interactions between static networks. "The strongest differential interactions are not necessarily those that are strong in static conditions, they are those that are most clearly changing" [4].

Most importantly, we begin with mapping the cognitive system and its conceptual space because since the conceptual space can be definition be conceptualized, having it to compare other functional state spaces to provides an analogy helpful in understanding each other system and its own functional state space. Take the hypothetical homeostasis system. While we can't immediately identify and enumerate the functional states in the homeostasis domain or the processes required to transition between those states, it is possible to identify that all the functional states in the cognitive domain are concepts, and we can roughly equate the number of concepts with the number of words we use. We can also roughly equate the number of processes in this conceptual domain with the number of reasoning processes that connect these concepts, which is a very large number. Similarly, if we identify a concept (word) with 
the category of domain object that represents functional states in the homeostasis domain, it might be possible to design programs to parse all available text describing such interactions to enumerate all known processes believed to be capable of transitioning between states of that category. In doing so it might be possible to begin to map out the homeostasis functional state space. Other techniques for mapping out such functional state spaces in order for those state spaces to be used as essentially a database for all models of all biological processes are continuing to emerge. For example, "as reconstructed biochemical reaction networks continue to grow in size and scope, there is a growing need to describe the functional modules within them. Such modules facilitate the study of biological processes by deconstructing complex biological networks into conceptually simple entities. The definition of network modules is often based on intuitive reasoning. As an alternative, methods are being developed for defining biochemical network modules in an unbiased fashion. These unbiased network modules are mathematically derived from the structure of the whole network under consideration" [5].

Human-Centric Functional Modeling combined with General Collective Intelligence also enables groups to collectively converge on the single understanding of biological systems that is most fit at describing observed behavior. In functional state space every theoretical model of any biological process is represented by a path between one functional state and another, that is, by a path through some functional state space, as is every implementation of every process.

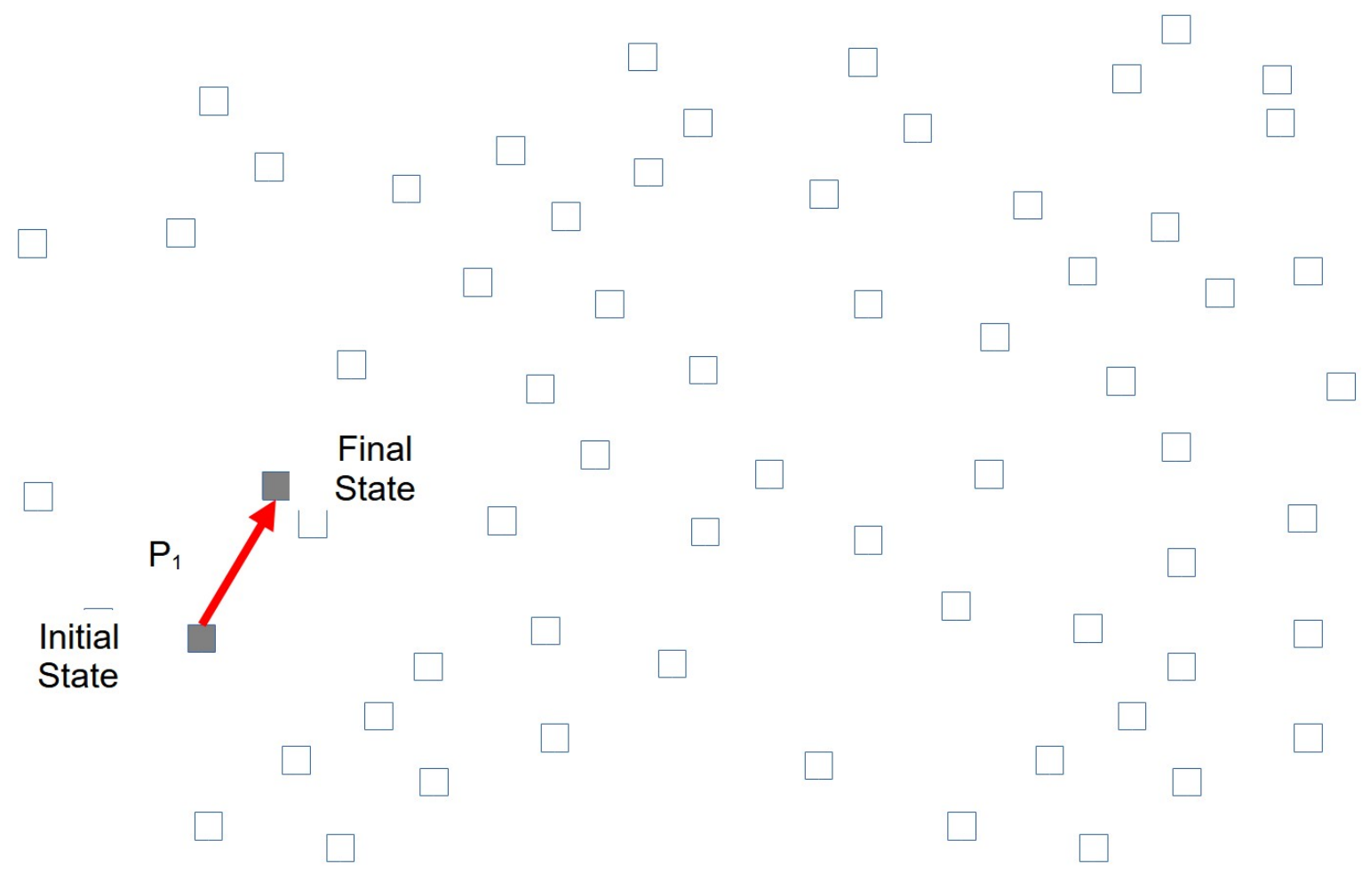

Figure 4. Every process executed by any model of a biological system can then potentially be represented as a path through a functional state space. 
By decoupling each model into a set of processes, those processes can be added to a library that can be used to increase general problem-solving ability in solving the problem of implementing functional models in each functional domain.

\section{Library of Processes}
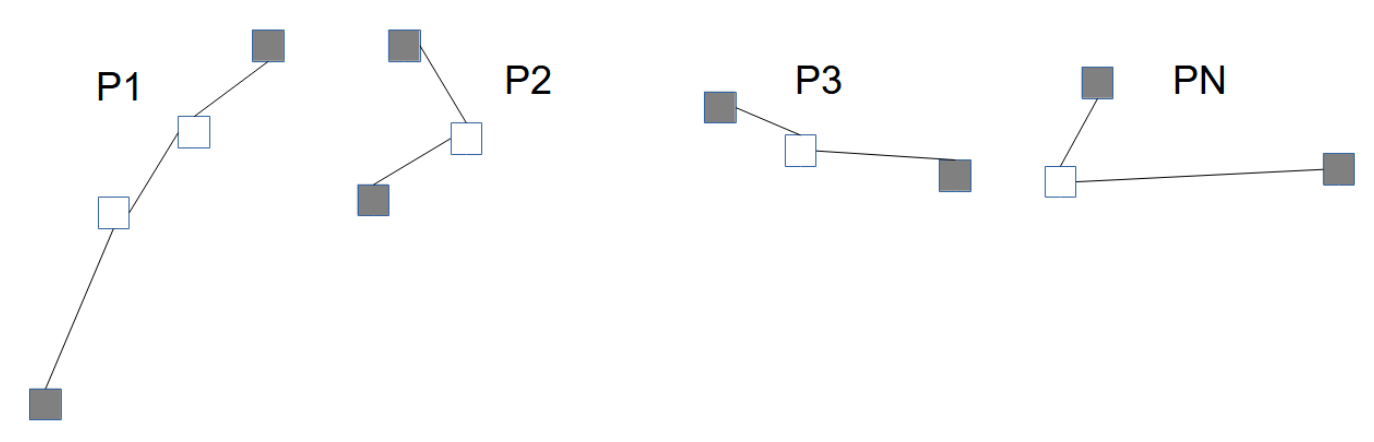

Figure 5. A library of theoretical models of processes as well as of implementations of processes.

We can then define a single measure of fitness by which all such processes might be compared.

\section{Fitness of Processes}

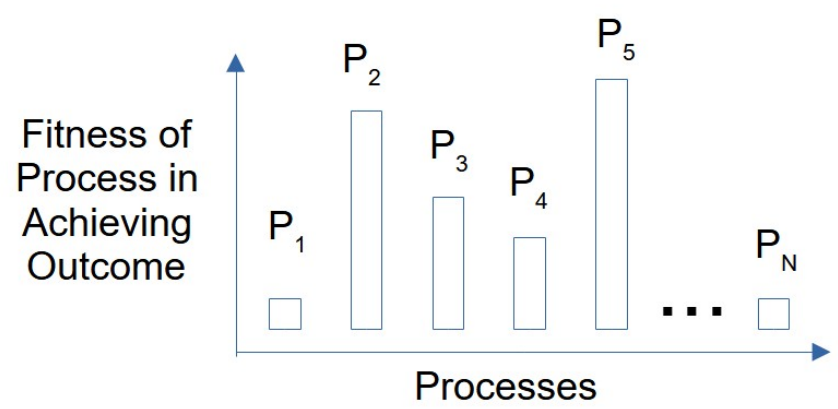

Figure 6. The fitness of each model of each process in a given context of execution can be compared.

This can enable each model to be reused in an exponentially greater number of instances where it is most fit in achieving an outcome, thereby exponentially increasing the group's collective ability to solve problems of understanding biological systems. 


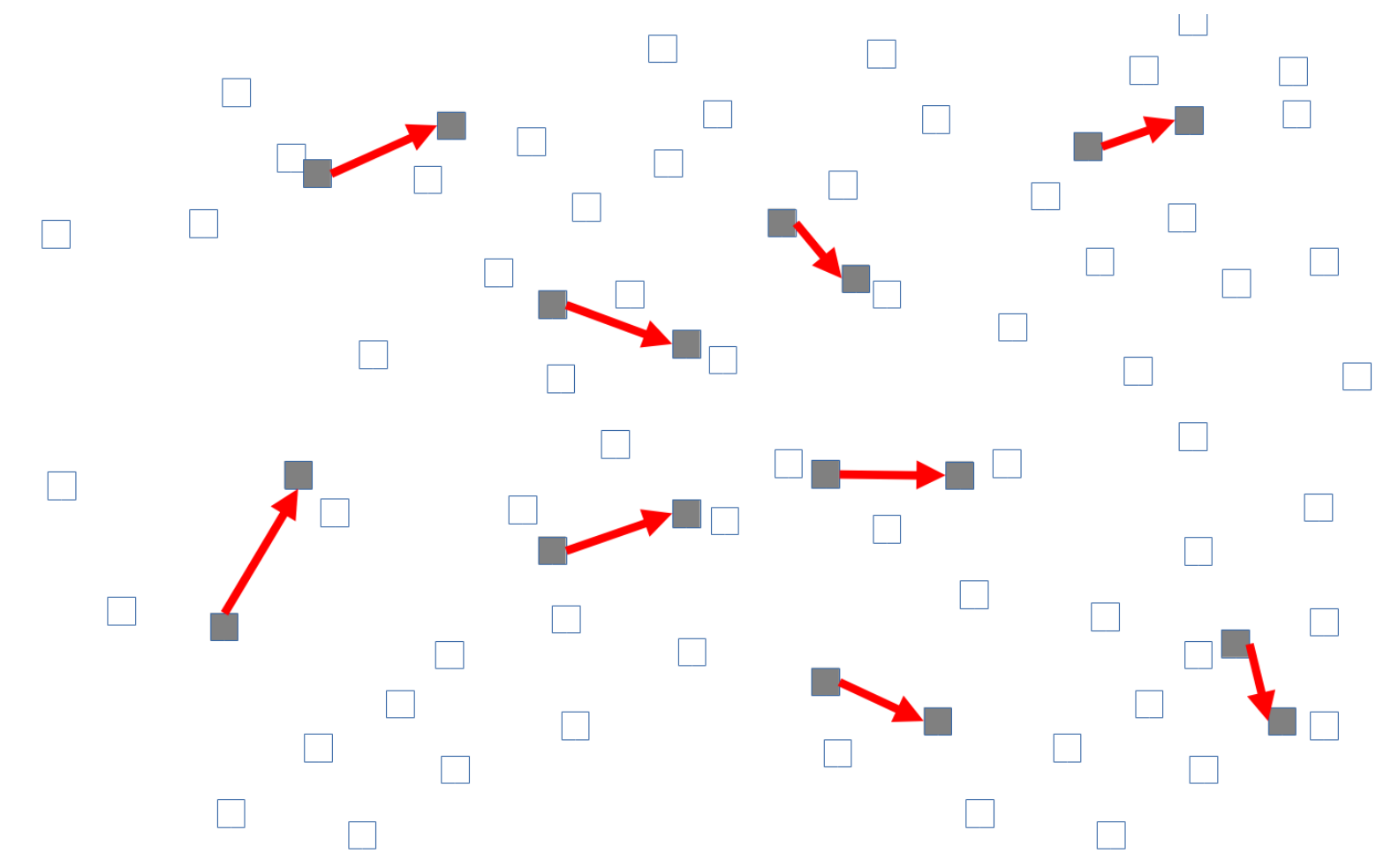

Figure 7. Reuse of the process model or implementation everywhere it applies.

\section{Future Directions}

Future work may explore the relationship between solving the same problem of stability in a given domain through achieving general problem-solving ability in that domain, and the relationship of that stability to any underlying thermodynamic principles, such as maximizing the production of entropy. Other potential areas of exploration are below:

- Protein Folding: Explore how a functional state space might be constructed using protein or complex protein groups as functional states, and using the addition or removal of individual or groups of amino acids as the processes that transform one protein into another. Explore how the folding structure of each protein in this functional state space can be determined by the relationship of that protein with other proteins in this space in terms of function.

- Gene Expression: If the assembly of a protein is represented by a path through a functional state space, then assembly of biological structures is a hierarchy of paths through that functional state space. Explore how the expression of genes or combinations of genes might be expressed as a hierarchy of paths through that functional state space. This problem has deep implications in General Collective Intelligence based smart manufacturing, in which product designs incorporate a hierarchy of component designs that have each been chosen so that they are reusable across entire industries in order to exponentially increase the economy of scale that comes with reuse, as well as other outcomes. This complex hierarchy of designs is stored in a "genome", and altering any part of this genome requires some "reproduction algorithm" that changes enough of the structure to impact the targeted outcome over time, but not too much so as to break genetic compatibility. Component designs that are reusable across entire industries are analogous to organic structures that are reused in different ways across an entire organism. This is one more example of how problems or even wicked problems [17] in human societies can potentially be generalized in some functional state space into problems that nature has already solved. 
- A Single Data Model at All Scales: Representing the functional states of each biological process as a network of nodes connected by edges representing all possible behaviors by which that biological process might change from one state to another, then if that functional state space is complete every functional model of every proposed implementation of every biological process must fit within that network of functional state spaces and the behaviors they contain. Solving the problem of defining a complete functional state space to represent biological processes is also then a solution to the problem of enabling all information about biological processes to be combined in a way that converges on a single deeper understanding. Analysis regarding how existing functional models of biological processes and their implementations fit into this Human-Centric Functional Modeling approach, and confirming that they do, has not yet been performed. Biologists might have great expertise in this area. Showing them that they have an interest in solving this problem as well can result in directing far more talent to problems that can impact a far larger number of people.

- Value Proposition of Human-Centric Functional Modeling: Creating the capacity to explore every possible relationship between every one of $\mathrm{N}$ biological processes is an exponential problem of order $\mathrm{N}$ that is not solvable by any previously existing technology. Describing behaviors in terms of functional state spaces, if the behaviors in those functional state spaces are "spanned" by a small number of functions, in the sense that all processes within that domain can be expressed as some composition of those functions, then this can exponentially increase the complexity of the behavior that can be experimentally probed within that domain. In terms of research, the combination of HCFM to provide a universal functional model of biological processes, and the use of General Collective Intelligence (GCI) to exponentially increase capacity to explore those relationships creates the potential for an exponential increase in capacity to understand biological processes and the complex relationships between them.

- Healthcare Benefits of Human-Centric Functional Modeling of Biological Organisms: Elaborating the application of HCFM to modeling the biology of plants or other organisms that might cause or be used to treat disease, or using it to model the entire human state space so that all medical data can be stored semantically, can exponentially increase capacity to achieve outcomes with healthcare and wellness interventions, as planned for the healthcare program in phase II of the Collective Intelligence based Program to Accelerate Achievement of the Sustainable Development Goals (CIPAA-SDGs) program.

- Verifying the Existence of Functional Domains within Organisms: The functional domains existing in organisms were deduced in terms of those required for a cell to display homeostasis as the basic property of life, then the ability to reproduce, then other abilities such as the ability to methodically change the stability of the cell in order to grow, the ability to methodically change reproduction so it is possible to evolve, the ability to methodically change the function of each cell so that multicellular cooperation is achievable, all the way up to the functional domains required for cognition, or for consciousness. Human-Centric Functional Modeling assumes that all biological processes can be represented as paths within some functional state space (i.e. as transitions from one functional state in that space to another). It remains to be confirmed experimentally whether biological organisms can be represented in terms of these functional domains, and whether all biological processes within those organisms can be represented as paths in some functional domain in this way.

- Evolutionary Origin of Functional Domains and the Formation of Systems: From the perspective of Human-Centric Functional Modeling a system is defined as an entity having behaviors that start with or end in functional states that are confined to the domain object of some functional state space. To consider how behavior might evolve to be confined to a functional state space, and in doing so develop into a "system", assume the functional components of the organism are cells. One cell might evolve a function. Through that function 
that cell might evolve to interact directly with another cell to accomplish some function $\mathrm{F}_{1}$. We'll call that a first order interaction. A cell might evolve to interact indirectly with some cell via another cell to accomplish some function $\mathrm{F}_{2}$. We'll call this a second order interaction. Yet another cell might evolve to interact via some third order interaction $\mathrm{F}_{3}$. Eventually these interactions might come to form a set that the organism is able to execute repeatably. At that point they form a system with behavior confined to a functional state space. However, the organism might not be able to detect interactions $\mathrm{F}_{\mathrm{N}}$ involving some number of cells $\mathrm{N}$ that is very large, thereby setting a limit on the complexity of interactions. Future directions might include exploring the limits to the complexity of interactions within each organism.

\section{Conclusions}

It has been stated that "we are confronted with problems that would have been unthinkable just a few years ago" and that the study of biomedical problems has become "difficult and "complex" [8]. As we move towards considering the whole rather than the components, systems thinking emerges as a "foundational underpinning of modern bioinformatics" [8]. It has also been stated that "molecular entities present in a cell (mRNA, proteins, metabolites,...) do not act in isolation, but rather in cooperation with each other to define an organism's form and function", and that "their concerted action can be viewed as networks of interacting entities that are active under certain conditions within the cell or upon certain environmental signals" [10]. A main challenge in systems biology is to model these networks, or in other words studying "which entities interact to form cellular systems or accomplish similar functions" [10]. Semantically modeling the human organism or any other organism is expected to radically increase collective capacity to address these issues, but is also itself a massive

task. Before this task can be achieved, it's important to first communicate the possibilities that might be presented by using Human-Centric Functional Modeling to achieve it, to encourage others to validate both the feasibility of the approach and its value proposition in this and other disciplines, until the value proposition appears to be present in a large enough range of disciplines to reliably motivate that effort.

\section{References}

[1] Newman MEJ (2010) Networks: an introduction. Oxford University Press, New York

[2] Andy E. Williams, Semantic Metrics Framework, Working paper (2021)

[3] Barabási, AL., Oltvai, Z. Network biology: understanding the cell's functional organization. Nat Rev Genet 5, 101-113 (2004). https://doi.org/10.1038/nrg1272

[4] Ideker, Trey, and Nevan J. Krogan. "Differential network biology." Molecular systems biology 8.1 (2012): 565.

[5] Jason A. Papin, Jennifer L. Reed, Bernhard O. Palsson, Hierarchical thinking in network biology: the unbiased modularization of biochemical networks, Trends in Biochemical Sciences, Volume 29, Issue 12, 2004, Pages 641-647, ISSN 0968-0004, https://doi.org/10.1016/j.tibs.2004.10.001.

[6] H. Arthur Woods, J. Keaton Wilson, An information hypothesis for the evolution of homeostasis, Trends in Ecology \& Evolution, Volume 28, Issue 5, 2013, Pages 283-289, ISSN 0169-5347, https://doi.org/10.1016/j.tree.2012.10.021.

[7] Swenson, R. (1989), Emergent attractors and the law of maximum entropy production: Foundations to a theory of general evolution. Syst. Res., 6: 187-197. https://doi.org/10.1002/sres.3850060302 
[8] Marcel Joly, Patrícia H.C. Rondó, The future of computational biomedicine: Complex systems thinking, Mathematics and Computers in Simulation, Volume 132, 2017, Pages 1-27, ISSN 0378-4754, https://doi.org/10.1016/j.matcom.2015.06.010.

[9] Helms, Michael, Swaroop Vattam, and Ashok Goel. "The effect of functional modeling on understanding complex biological systems." International Design Engineering Technical Conferences and Computers and Information in Engineering Conference. Vol. 44137. 2010.

[10] Lore Cloots, Kathleen Marchal, Network-based functional modeling of genomics, transcriptomics and metabolism in bacteria, Current Opinion in Microbiology, Volume 14, Issue 5, 2011, Pages 599607, ISSN 1369-5274, https://doi.org/10.1016/j.mib.2011.09.003

[11] Strumia, Alberto. From Fractals and Cellular Automata to Biology: Information as Order Hidden Within Chance. 2020.

[12] Havlin, Shlomo, et al. "Fractals in biology and medicine." Chaos, Solitons \& Fractals 6 (1995): 171-201.

[13] Andy E. Williams, A Revolution in Systems Thinking?, Proceedings of the World Organization of Systems and Cybernetics (WOSC) 2021 Congress, Accepted (2021), link

[14] Williams, A. E. (2021, October 2). Using Systems Thinking to Exponentially Increase Impact. In review (2021), Retrieved from osf.io/preprints/africarxiv/dcjyf

[15] Williams, A. E., Defining a Continuum from Individual, to Swarm, to Collective Intelligence, to General Collective Intelligence, International Journal of Collaborative Intelligence, in press (2021)

[17] Andy E. Williams, Are Wicked Problems a Lack of General Collective Intelligence?, AI \& Society, DOI: 10.1007/s00146-021-01297-8, In print (2021)

[18] Williams, A. E. (2021, January 11). AI Safety and General Collective Intelligence. https://doi.org/10.31730/osf.io/gw3ks 